\title{
Rate-Dependent Adhesion between Opposed Perfluoropoly(alkyl ether) Layers: Dependence on Chain-End Functionality and Chain Length
}

\author{
Marina Ruths and Steve Granick* \\ Department of Materials Science and Engineering, University of Illinois, \\ Urbana-Champaign, Urbana, Illinois 61801 \\ Received: February 27, 1998
}

\begin{abstract}
Adhesion was measured repeatedly between opposed molecularly thin layers of perfluoropoly(alkyl ether)s on mica, with particular attention to the dependence of the adhesion on chain-end functionality and chain length of the polymer. The polymer layers were kept in contact for specified times that differed by 4 orders of magnitude $(0.01-500 \mathrm{~s})$ and then unloaded (separated) at rates that varied by 5 orders of magnitude $(0.003-$ $120 \mu \mathrm{m} / \mathrm{s}$ of a detachment device with spring constant $1.1 \times 10^{4} \mathrm{~N} / \mathrm{m}$ ) using a modified surface forces apparatus with piezoelectric actuators supporting one surface. Rate dependence resulting in an increase of the adhesion by a factor up to 3 was observed when the unloading rate exceeded a critical value that was found to depend not only on the polymer sample but also on the contact time between opposed layers. At a given unloading rate, the adhesion was larger, the longer the surfaces were left in stationary contact before separation. No history dependence was observed when the unloading rate was raised and subsequently lowered. For relatively short contact times $(0.01-1 \mathrm{~s})$, the critical unloading rate was larger for the polymer that contained polar end groups than for unfunctionalized polymer of similar chain length, indicating a lower mobility in the former case, and thus slower initial interdigitation of the chains, since the polar end group had a preferential affinity for the underlying surface. This effect vanished for contact times of 100-500 s. For polymer chains without polar end groups, the critical value of the unloading rate was smaller, the larger the chain length, and the increase in adhesion with unloading rate was more pronounced, presumably reflecting slower relaxation of the longer chains. The rate dependence is analyzed quantitatively.
\end{abstract}

\section{Introduction}

Traditional explanations of adhesion emphasize the work needed to overcome equilibrium interactions (potential energy as it depends on surface separation), but it is commonly observed that adhesion increases with separation rate. This has been noticed independently in several disciplines with conflicting explanations: in the study of bioreceptor interactions, where it has been attributed to rate-dependent shifts in the dependence of potential energy on surface separation, ${ }^{1,2}$ and in polymer adhesion, where it has been attributed in some instances to pullout of chains that had become entangled in opposed polymer networks ${ }^{3,4}$ but in most cases to energy dissipation within the bulk of the polymer rather than at its surface. ${ }^{5-9}$ Some approaches to analyze rate dependence separate the adhesion into the product of a term proportional to the surface energy and a second term proportional to viscoelastic losses in the underlying bulk material. ${ }^{5-7}$ The best-developed and most widely used approaches to describe adhesion, such as the classical "JKR" theory of Johnson, Kendall, and Roberts, ${ }^{10}$ are based on a balance between potential energy, surface energy, and elastic deformation within the solid bodies. Dissipation and rate dependence are ignored in this line of analysis.

If the adhesion is too high when solid bodies begin to move past one another, wear and material degradation ensue. This is a major cause of hard disk failure in personal computers and other information storage devices. ${ }^{11}$ Similar problems arise in micromotors, known as microelectromechanical systems (MEMS), ${ }^{12}$ and even in the internal combustion engine during startup. ${ }^{13}$ To minimize adhesion, a common strategy is to coat the solid surfaces with an organic monolayer or "friction modifier". ${ }^{14}$ In such instances the predominant source of dissipation cannot be attributed to well-known effects of dissipation in the underlying support material; ${ }^{-9}$ when a thin liquid film coats a hard elastic substrate, the predominant energy dissipation occurs at the surface, within the liquid film itself. In the present study we are concerned with direct measurements of adhesion between elastic bodies as modified by interactions within molecularly thin organic films that coat the contacting surfaces. In this case, rate dependence can be attributed to dissipation within layers a single molecule thick.

Many experimental studies of such systems are rendered ambiguous by uncertainty concerning the perfection of surfacesurface contact: if the surfaces are rough, the actual area of contact is much less than the nominal area of contact but might increase during the course of an experiment. ${ }^{15,16}$ This difficulty is avoided in experiments on spherical caps of elastomeric polymers in contact with an opposing, flat surface; ${ }^{17-19}$ the soft elastomer conforms to the opposed surface, and molecular contact is achieved. Some remaining experimental difficulties include time effects on the deformations of the relatively large and soft bodies studied, ${ }^{19}$ which may complicate the interpretation of results on adhesion hysteresis and pull-off forces. The technique has been extended to studies of the effects of contact time and separation rate on the adhesion between an elastomer cap and a surface covered with grafted polymer chains that penetrate into the elastomer network. ${ }^{3,4}$ Theoretical models ${ }^{20,21}$ for such systems, based on the elastic energy and contact area of the penetrating chains being pulled out of the network, predict 
a linear increase in apparent adhesion or "interfacial toughness" as the separation rate is increased. This has been observed experimentally in some studies, ${ }^{3}$ while in other experiments, ${ }^{4}$ the response was found to be strongly nonlinear. A limitation is that when viscoelastic or time-dependent observations are obtained on these systems, it is ambiguous whether the result is an effect of events at the surface or within the underlying bulk material.

Alternatively, molecularly smooth solid surfaces can be used to study adhesion experimentally. The surface force apparatus (SFA) technique ${ }^{22}$ has been used successfully to study adhesion between surfaces separating both in air and in liquids. The time effects ${ }^{19}$ arising from the not always uniform properties of relatively large elastomer spheres are generally avoided in the SFA, where the interacting thin surface layers are supported on a more rigid mica and glue layer on a silica disc. The contact between the half-cylinders in the SFA can be thought of as one single asperity, and molecular contact between adsorbed layers can be achieved instantly over a macroscopic area (diameter $\sim 50-100 \mu \mathrm{m})$ if a smooth enough surface layer with a welldefined thickness can be formed. The solid supports themselves obey the principles of linear elasticity and therefore the solid fracture mechanics commonly employed to analyze adhesive unloading. ${ }^{10,23}$ Therefore, when rate dependence is observed in a experiment, its origin lies in relaxations at the contact interface. Potentially, there is the possibility to adopt an approach more molecular than is usual in fracture mechanics to relate adhesion unloading to viscoelastic events within interfacial layers.

In this paper we describe experiments in which the unloading (separation) rate between opposed molecularly smooth surfaces was varied by many orders of magnitude with independent control of the stationary contact time before separation. The range of both unloading rate and of stationary contact time greatly exceed those possible in conventional loading-unloading "JKR" experiments. ${ }^{17-19,24}$ In a previous paper, we have studied rate-dependent adhesion between surfactant and block copolymer films that were rigidly localized to the underlying solid surfaces and found pronounced rate dependence. ${ }^{25}$ The main new result of the present paper is to confirm the generality of the previous observations and to extend them to the case where the molecules are not strongly anchored to the surface. We also demonstrate the strong effects of polymer chain-end functionality and chain length.

The surfaces studied were thin, physisorbed layers of four different linear perfluoropoly(alkyl ether)s. Perfluoropoly(alkyl ether)s are very stable against oxidation and therefore useful not only for hard-disk lubrication ${ }^{11,26-31}$ but also as hightemperature gas turbine oils. ${ }^{32}$ Furthermore, since they are liquid in a wide range of temperatures and have very low volatility, they are used for lubrication in aerospace applications and in laboratory ultrahigh-vacuum equipment. ${ }^{32}$ We find the adhesive unloading forces between adsorbed, thin layers of these polymers to be decidedly rate- and time-dependent.

\section{Experimental Methods and Materials}

The pull-off force, $F$, needed to separate two surfaces after different times in contact, was measured with a modified surface forces apparatus. ${ }^{33,34}$ Piezoelectric bimorph elements, attached to a double-cantilever spring supporting the lower surface, were used to vary the separation rate of the surface supports. By leading a programmed waveform from a function generator to the piezoelectric bimorph device, two mica surfaces coated with polymer were brought together at a constant rate (no dependence

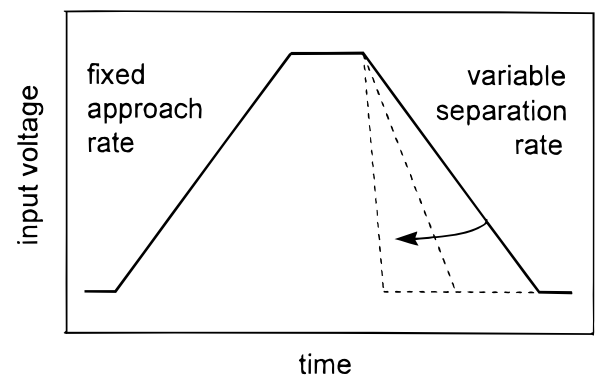

Figure 1. Schematic diagram of the programmed waveform and the resulting positioning of the surface supported by the piezoelectric bimorph device in the surface forces apparatus as function of time. The surfaces first approach each other at a chosen approach rate, are held stationary for $0.01-500 \mathrm{~s}$, and are then separated by displacing a spring at a rate chosen in the range $0.003-120 \mu \mathrm{m} / \mathrm{s}$.

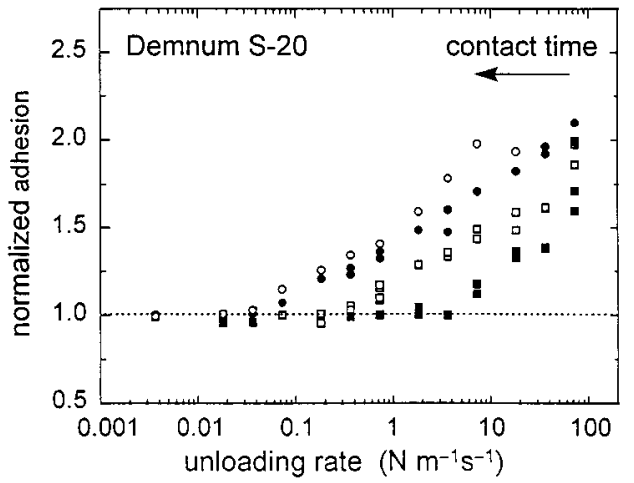

Figure 2. Normalized adhesion $\left(F / F_{0}\right.$ or $\left.\gamma_{\text {eff }} / \gamma_{0}\right)$ for unfunctionalized Demnum S-20 $\left(M_{\mathrm{w}}=2500 \mathrm{~g} / \mathrm{mol}\right)$ plotted as a function of logarithmic unloading rate, $\Delta\left(F_{\mathrm{s}} / R\right) / \Delta t$. Contact times in Figures 2-5: (四) 0.01, () $1,(\bullet) 100$, and (O) 500 s. $\gamma_{0}$ is described in the text and in Table 2 .

on this rate was observed in our experiment, since the surfaces spontaneously jumped into contact from a small separation), kept in contact for a controlled time, and then separated at controlled, constant rates. The deflection of the device is shown schematically in Figure 1. The mechanical distance controls ${ }^{22}$ and the multiple-beam interference technique ${ }^{35}$ used to measure adsorbed layer thickness and surface separation in a standard surface forces apparatus were retained in our experimental setup. The optical technique allows measurements of the thickness of the adsorbed layers (relative to calibrations of uncoated pieces of the same mica) with an accuracy of $1-2 \AA$. Since the contact area of the interacting surfaces varied slightly between different experiments and between different contact points between surfaces in the same experiment, the pull-off forces were normalized by the radius of curvature of the undeformed surfaces, $R$ (generally $1.5-2 \mathrm{~cm}$ ).

The unloading rate produced by applying a negative load with the double-cantilever bimorph-spring assembly described above was expressed as the rate of change in separation force, $\Delta\left(F_{\mathrm{s}}\right)$ $R) / \Delta t$. Since the spring constant of the device was $k=1.1 \times$ $10^{4} \mathrm{~N} / \mathrm{m}$ (measured from its resonance frequency), the unloading rates in Figures 2-5 correspond to a vertical separation rate of $0.003-120 \mu \mathrm{m} / \mathrm{s}$. For comparison with theories based on analysis of the crack velocity (separation rate in the lateral direction) during separation, ${ }^{7,8}$ it is also relevant to know this latter quantity. As described in an earlier report, ${ }^{25}$ we estimate that for this spring stiffness the average lateral separation rate during separation was approximately 10-12 times larger than the device separation rate in the direction normal to the surfaces. The experimental technique and the relation between lateral and vertical separation rates were described in detail in ref 25 . 

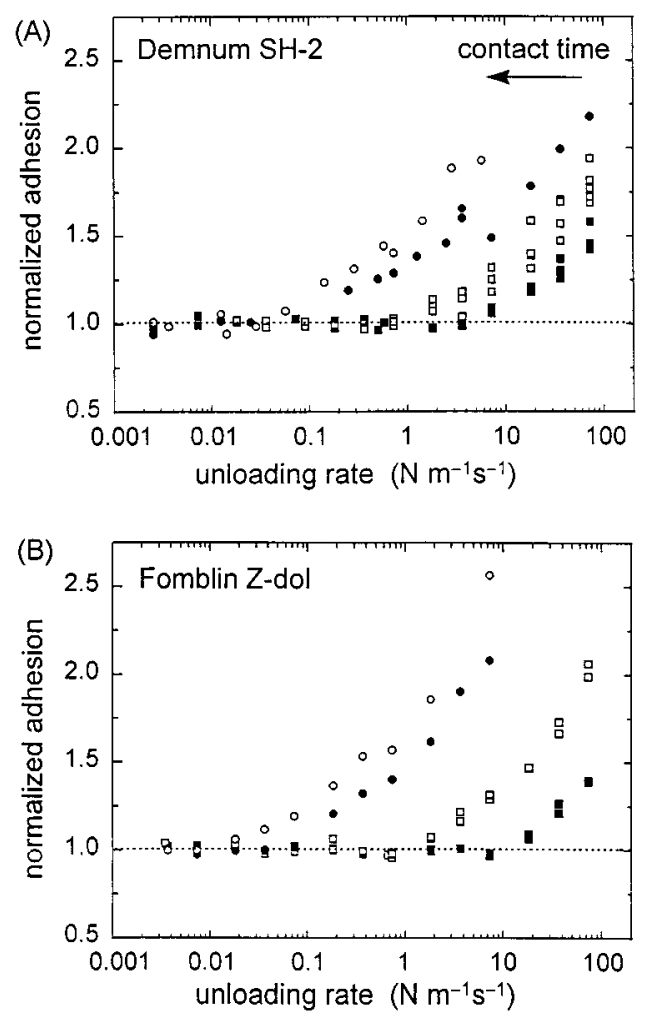

Figure 3. Effects of polar end groups: normalized adhesion for $(\mathrm{A})$ Demnum SH-2 $\left(M_{\mathrm{w}}=3200 \mathrm{~g} / \mathrm{mol}\right)$ and $(\mathrm{B})$ Fomblin Z-dol $\left(M_{\mathrm{w}}=\right.$ $3100 \mathrm{~g} / \mathrm{mol}$ ), plotted as a function of logarithmic unloading rate, $\Delta\left(F_{\mathrm{s}} / R\right) / \Delta t$, as described in Figure 2. Symbols as in Figure 2.

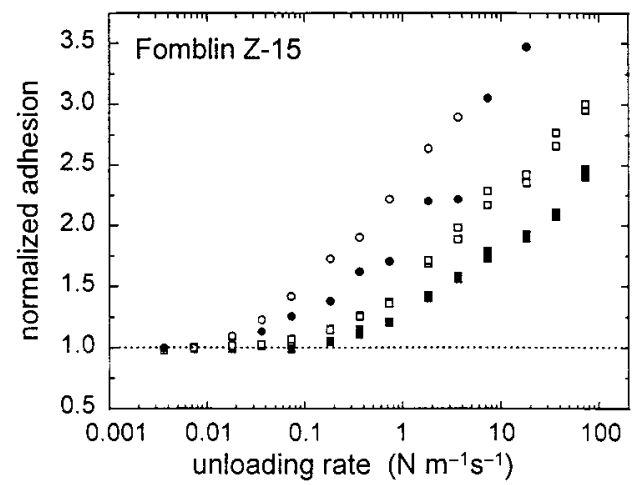

Figure 4. Effects of molecular weight: normalized adhesion for unfunctionalized Fomblin Z-15 $\left(M_{\mathrm{w}}=16700 \mathrm{~g} / \mathrm{mol}\right)$ plotted as a function of logarithmic unloading rate, $\Delta\left(F_{\mathrm{s}} / R\right) \Delta t$, as described in Figure 2. Symbols as in Figure 2.

Back-silvered mica sheets were glued onto half-cylindrical fused silica discs with a 1:1 mixture of dextrose and galactose. Thin films of different perfluoropoly(alkyl ether)s (Table 1) were allowed to adsorb onto the mica sheets by immersing the whole discs in $10-12 \mathrm{~g}$ of $0.1 \%(\mathrm{w} / \mathrm{w})$ polymer solution in perfluoroheptane for $2 \mathrm{~h}$ at room temperature. Three of the polymers we have studied had a similar, relatively small chain length and differed mainly in their chain end groups: either unfunctionalized $\left(-\mathrm{CF}_{3}\right)$, or one end group being a $p-$ phenoxyanilinium salt of a carboxylic acid or both end groups being hydroxylated $\left(-\mathrm{CH}_{2} \mathrm{OH}\right)$. An unfunctionalized polymer of longer chain length was also studied. The perfluorinated polymers were used as received. The perfluoroheptane (Fluka, approximately $80 \%$ perfluoro- $n$-heptane) was dried over molecular sieves (Aldrich, pore size $5 \AA$ ) overnight and filtered through a Millex-SR filter (Millipore, pore size $0.5 \mu \mathrm{m}$ ) before preparing the polymer solutions. It was established that the adsorption time of $2 \mathrm{~h}$

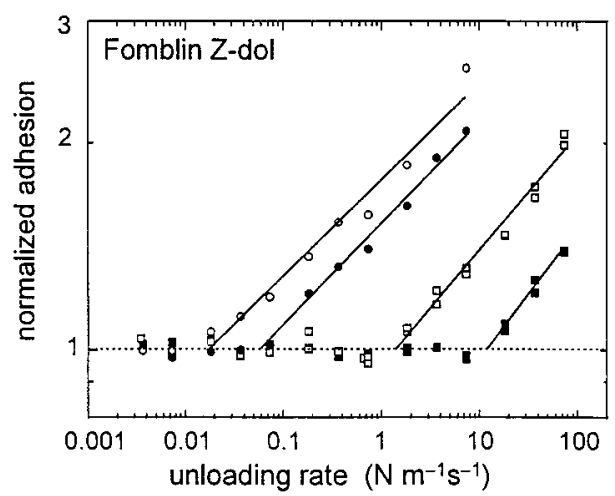

Figure 5. Normalized adhesion for Fomblin Z-dol (the data in Figure 3B) plotted on $\log -\log$ scales. The fits used to determine the critical unloading rate for each contact time (shown in Figure 6 for all four polymer systems) are described in the text, and the average linear slope of the fits, $B_{\text {ave }}$, for each system is given in Table 2 .

was enough to obtain a constant thickness of the adsorbed layers of each polymer (Table 2), since the same thicknesses were obtained also after longer adsorption times of up to $18 \mathrm{~h}$. After the adsorption, the discs with the polymer-coated mica were lifted out of the solution (with the mica surface in a horizontal position) and transferred while still wet to a container with approximately $10 \mathrm{~g}$ of dried and filtered perfluoroheptane, where they were kept for $1 \mathrm{~h}$ to remove possible excess of polymer.

The discs were then removed from the rinsing solvent and blown dry with $\mathrm{N}_{2}$ gas. They were mounted in the surface forces apparatus, which was purged with $\mathrm{N}_{2}$ gas for approximately $16 \mathrm{~h}$ to further remove any trace of solvent. During the measurements of distances and pull-off forces, the purging was stopped, and a tray with $\mathrm{P}_{2} \mathrm{O}_{5}$ was placed in the chamber of the instrument to take up moisture. All measurements were done at $25{ }^{\circ} \mathrm{C}$.

\section{Results}

Thickness of the Adsorbed Layers. For all four systems, the perfluorinated poly(alkyl ether)s were found to adsorb from perfluoroheptane to form thin, smooth layers with a thickness of approximately $12 \AA$ on each mica surface (summarized in Table 2) as measured by multiple-beam interferometry for two polymer-coated surfaces in adhesive contact. Since perfluoroheptane is a good solvent for Fomblin and Demnum perfluoropoly(alkyl ether)s at room temperature, ${ }^{36}$ the driving force for the physisorption of these nonpolar molecules to the interface between the polar mica and the nonpolar solvent is likely the difference in their dielectric properties: ${ }^{37}$ The refractive indexes of mica, Demnum S-20, and perfluoroheptane at $25{ }^{\circ} \mathrm{C}$ are $1.58-1.60,{ }^{37} 1.290,{ }^{38}$ and $1.259,{ }^{39}$ respectively. The polymers containing polar functional groups are expected to have an additional affinity for the polar surface, ${ }^{27-31}$ but no difference in adsorbed layer thickness was observed for these samples. The layer thickness of $12 \AA$ corresponds to not more than $2-3$ segmental diameters of the perfluoropoly(alkyl ether), since the cross-sectional area of a $-\mathrm{CF}_{2}-$ chain ${ }^{40}$ is $28.3 \AA^{2}$ and the chain diameter of the Fomblin backbone has been estimated to be around $7 \AA .{ }^{27}$ For the three samples of short chain length, the film thickness is close to their hydrodynamic radius in solution, $9-10 \AA,{ }^{36,41}$ while the film thickness for the sample of longer chain length is approximately $1 / 2$ its hydrodynamic radius of $22 \AA .{ }^{36,41}$ In adhesive contact, the interferometry showed a homogeneous contact zone, indicating that the surface roughness is within the experimental thickness resolution of $1-2 \AA$. 
TABLE 1: Properties of the Perfluoropoly(alkyl ether) Samples

\begin{tabular}{|c|c|c|c|c|}
\hline trade name & chemical formula & composition & $M_{\mathrm{w}}(\mathrm{g} / \mathrm{mol})$ & $M_{\mathrm{w}} / M_{\mathrm{n}}{ }^{d}$ \\
\hline Demnum S-20a & $\mathrm{CF}_{3} \mathrm{CF}_{2} \mathrm{O}\left(\mathrm{CF}_{2} \mathrm{CF}_{2} \mathrm{CF}_{2} \mathrm{O}\right)_{n} \mathrm{CF}_{2} \mathrm{CF}_{3}$ & $n \approx 14$ & 2500 & $1.1-1.2^{36}$ \\
\hline $\begin{array}{l}\text { Demnum } \mathrm{SH}-2^{b} \\
\text { ( } p \text {-phenoxyanilinium } \\
\text { salt of Demnum-SH) }\end{array}$ & $\mathrm{F}\left(\mathrm{CF}_{2} \mathrm{CF}_{2} \mathrm{CF}_{2} \mathrm{O}\right)_{n} \mathrm{CF}_{2} \mathrm{CF}_{2} \mathrm{COO}^{-} \mathrm{N}^{+} \mathrm{H}_{3} \mathrm{C}_{6} \mathrm{H}_{4} \mathrm{OC}_{6} \mathrm{H}_{5}$ & $n \approx 17$, functionality $75 \%$ & 3200 & 1.05 \\
\hline Fomblin Z-dolc ${ }^{c}$ & $\mathrm{HO}-\mathrm{CH}_{2} \mathrm{CF}_{2} \mathrm{O}\left(\mathrm{CF}_{2} \mathrm{CF}_{2} \mathrm{O}\right)_{m}\left(\mathrm{CF}_{2} \mathrm{O}\right)_{n} \mathrm{CF}_{2} \mathrm{CH}_{2}-\mathrm{OH}$ & $m \approx n \approx 16$ & 3100 & $<1.05$ \\
\hline Fomblin Z-15 ${ }^{c}$ & $\mathrm{CF}_{3} \mathrm{O}\left(\mathrm{CF}_{2} \mathrm{CF}_{2} \mathrm{O}\right)_{m}\left(\mathrm{CF}_{2} \mathrm{O}\right)_{n} \mathrm{CF}_{3}$ & $m / n \approx 2 / 3 \approx 77 / 116$ & 16700 & $<1.05$ \\
\hline
\end{tabular}

${ }^{a}$ Daikin Industries Ltd., donated by S. Sharma and H. Paige, Wright Laboratory, Wright Patterson Air Force Base, OH. ${ }^{b}$ Donated by A. Koike, Hitachi Research Laboratories, Ibaraki, Japan. ${ }^{c}$ Ausimont USA, Inc., donated by H. Brown and D. Yoon, IBM Almaden Research Center, San Jose, CA. ${ }^{d} M_{\mathrm{w}}$ and $M_{\mathrm{n}}$ denote the weight-average and number-average molecular weight, respectively.

TABLE 2: Experimental Results for the Adsorbed Polymer Layers

\begin{tabular}{cccccc}
\hline trade name & $\begin{array}{c}\text { thickness } \\
(\AA)\end{array}$ & $\begin{array}{c}-(F / R)_{0} \\
(\mathrm{mN} / \mathrm{m})\end{array}$ & $\begin{array}{c}\gamma_{0}=-(F / R)_{0} / 3 \pi \\
(\mathrm{mN} / \mathrm{m})\end{array}$ & $\begin{array}{c}\gamma_{0}=-(F / R)_{0} / 4 \pi \\
(\mathrm{mN} / \mathrm{m})\end{array}$ \\
\hline Demnum S-20 & $13 \pm 1$ & $360 \pm 10$ & $38 \pm 1$ & $29 \pm 1$ & $0.105 \pm 0.008$ \\
Demnum SH-2 & $11 \pm 1$ & $360 \pm 10$ & $38 \pm 1$ & $29 \pm 1$ & $0.12 \pm 0.01$ \\
Fomblin Z-dol & $12 \pm 1$ & $360 \pm 10$ & $38 \pm 1$ & $29 \pm 1$ & $0.16 \pm 0.02$ \\
Fomblin Z-15 & $12 \pm 1$ & $400 \pm 10$ & $42 \pm 1$ & $32 \pm 1$ & $0.16 \pm 0.02$
\end{tabular}

Approach and Separation. A schematic diagram, showing an approach of the opposed surfaces, pause (surfaces stationary at constant separation) for a specific time, and subsequent unloading, is given in Figure 1. As the polymer-covered surfaces were brought toward each other at a constant, slow approach rate $(1.2 \mu \mathrm{m} / \mathrm{s})$ using a programmed waveform, attraction was observed starting below a separation of approximately $80 \AA$ between the outer surfaces of the adsorbed polymer layers. The surfaces then spontaneously jumped into a flattened, adhesive contact $(D=0$, total film thickness around $24 \AA$ ) from a separation of approximately $30 \AA$. This behavior is consistent with the effects of van der Waals attraction acting between the surfaces, as has been observed previously in studies of different smooth polymer and surfactant layers separated by air. ${ }^{18,24}$ The separation of the surfaces before the approach was adjusted so that no additional pressure was applied after the surfaces had spontaneously jumped into contact.

As the surfaces were separated at a chosen, constant rate, the radius of the flattened contact area was found to decrease from its initial value of approximately $30 \mu \mathrm{m}$ until it became too small to be conclusively measured by eye (around $1-2 \mu \mathrm{m}$ ), and finally the surfaces jumped apart. The thickness of the confined film remained constant during this decrease of the contact area. A similar separation from a small contact area (far smaller than would be expected from the classical JKR theory $\left.{ }^{10}\right)$ was also observed for layers of diblock poly(2vinylpyridine)-polybutadiene copolymer, but not for glassy polymer layers or anchored, short chains such as adsorbed surfactant monolayers, ${ }^{25}$ where the separation occurs from a finite contact area (separation contact radius $\approx 0.5-0.6 \times$ initial contact radius) as expected from the JKR theory. At this time, we are unsure of the reason for these systematic differences, which seem to be correlated with the degree of local segmental mobility in different adsorbed thin films. The adhesion measurements were repeatable for dozens of cycles of approach and separation. No history dependence was observed when the separation rate was raised and subsequently lowered.

Absence of Capillary Forces. Based on "stiction" measurements of surfaces with well-defined asperities (craters with a diameter of a few micrometers), it has been suggested that 15$30 \AA$ thick Fomblin Z-dol films behave like a liquid even when thermally bonded to the surface and that a meniscus is formed around the points of contact between the asperities and the sliding head of the instrument. ${ }^{42}$ It has also been suggested that a meniscus was formed between $30 \AA$ thick Fomblin Z03 and Z-dol films on a silicon oxide surface and an atomic force microscope (AFM) tip. ${ }^{27}$ However, such effects-the resulting formation (and breakage) of a meniscus on separation-should be readily detectable with our optical technique. For the present very thin films on molecularly smooth mica substrates, we observed neither meniscus nor change in thickness (within the experimental accuracy of $1-2 \AA$ ) in or around the contact area at any contact time or unloading rate studied. Nor did we observe pullout of material during separation.

In this context, it is relevant to discuss the lateral mobility in thin films of perfluorinated polymers on various substrates. The lateral diffusion (initial spreading) of polyperfluoropropylene oxides on $\mathrm{SiO}_{2}$ and on gold surfaces has been studied by scanning microellipsometry. ${ }^{26}$ The migration of this polymer was found to be independent of film thickness below $10 \AA$, since the transport then becomes controlled by surface diffusion. ${ }^{26}$ The results imply a surface diffusion coefficient of ca. $2 \times 10^{-11} \mathrm{~m}^{2} / \mathrm{s}$ at a film thickness of $10 \AA$ for $M_{\mathrm{w}}=3000$ $\mathrm{g} / \mathrm{mol}$, where $M_{\mathrm{w}}$ denotes weight-average molecular weight. ${ }^{26}$

Similar results have been obtained with the same technique for the spreading of 40-50 $\AA$ thick Fomblin Z-dol and unfunctionalized Fomblin $\mathrm{Z}$ films on native silicon oxide surfaces and on thermally bonded Fomblin Z-dol. ${ }^{28,29}$ At $0 \%$ humidity the Fomblin $Z\left(M_{\mathrm{w}}=5400 \mathrm{~g} / \mathrm{mol}\right)$ showed diffusionlike spreading on silicon oxide with a surface diffusion coefficient around $10^{-10} \mathrm{~m}^{2} / \mathrm{s}$, while Fomblin Z-dol $\left(M_{\mathrm{w}}=3100\right.$ $\mathrm{g} / \mathrm{mol}$ ) formed a steplike profile with a step height comparable to the radius of gyration, $R_{\mathrm{g}}$, of the polymer. ${ }^{28}$ The "step" layer had a diffusion coefficient of $(4-5) \times 10^{-11} \mathrm{~m}^{2} / \mathrm{s}$. (The differences compared to polyperfluoropropylene oxides ${ }^{26}$ may possibly be due to differences in film thickness.) However, on a layer of thermally bonded Z-dol, none of the perfluorinated polymers studied spread even after several days, but, similar to the behavior of physisorbed Fomblin Z-dol during initial spreading (i.e., the "step" profile), appeared to dewet. ${ }^{28}$ The spreading of perfluorinated polymers on an already formed layer is thus orders of magnitude slower than the initial spreading on a polar surface.

The relevance to our present experiments is that if the polymer were to flow along the surface to form a meniscus or become pulled up to form a "lump" at the point of separation, the respreading over the contact area (radius approximately $30 \mu \mathrm{m}$ ) would be very slow, and we should expect differences between the pull-off forces measured at the first and subsequent separations in our experiments. We should also expect an increasing film thickness. These phenomena were not observed, and for these reasons, a significant contribution of meniscus 
breakup to the adhesion measured in these experiments can likely be discounted.

Adhesion. Control experiments described in our previous paper showed no rate dependence for the adhesion between either close-packed monolayers of self-assembled chains or glassy polymers. ${ }^{25}$ When rate dependence was observed, it could be ascribed to disengagement of organic monolayers that had become partially interdigitated during the time in contact before separation forces were applied. ${ }^{25}$

For the present perfluoropoly(alkyl ether) samples, we observed classical rate-independent adhesion provided that the unloading rate was slow, but a significant increase in the pulloff force, by a factor up to 3 , when the unloading rate exceeded a critical level. In addition, the adhesion was larger, at a given unloading rate, the longer the surfaces were left in stationary contact before separation. The constant pull-off force measured at slow unloading rates was normalized by the mean radius of curvature of the undeformed surfaces, and this limiting value, $(F / R)_{0}$, is tabulated for the four polymer samples in Table 2 . (The pull-off force is attractive and thus negative by definition.)

Rate-dependent pull-off forces, measured at unloading rates above a critical value for each contact time, were normalized by the constant pull-off force at slow rates for two reasons: first, to eliminate systematic errors introduced by uncertainty in the radius of curvature measured in independent experiments, and, second, to circumvent uncertainty regarding the proper method by which to infer surface energy from pull-off force in the case of rate-dependent adhesion (see discussion below). These normalized values of pull-off force are plotted as functions of the unloading rate (rate of change in separation force), $\Delta\left(F_{\mathrm{S}} / R\right) / \Delta t$ (see the Experimental Section), in Figures 2, 3A, 3B, and 4 for Demnum S-20, Demnum SH-2, Fomblin Z-dol and Fomblin Z-15, respectively.

\section{Discussion}

Interdiffusion of Contacting Layers. The increased adhesion with increasing contact time at a chosen unloading rate (Figures 2-4) suggests that interdigitation of the two polymer layers developed continuously during the investigated times of stationary contact. It is natural to inquire into the quantitative shifts of our data with elapsed time in contact, since the length of diffusive interpenetration between layers might in the simplest approach be expected to scale with $t^{1 / 2}$, where $t$ is the contact time. Such a dependence has been predicted ${ }^{43}$ and observed ${ }^{44}$ for the interdiffusion of polymer chains to the point of entanglement with subsequent chain pullout. The bulk mutual diffusion coefficient for polymers well above their bulk glass transition temperature $\left(T_{\mathrm{g}}\right)$, is, however, orders of magnitude too large to explain our observation of slowly developing adhesion between these thin films. At bulk diffusion rates, interdiffusion to an interfacial width of the thickness of our whole sample (around $24 \AA$ ) would occur already at the shortest contact time $(0.01 \mathrm{~s})$ and fastest unloading rate studied. The adsorbed layers in our experiments are, however, very thin (approximately $12 \AA$ on each mica surface), and the interdiffusion process cannot be expected to be the same as in the bulk. The slow development of the adhesion with contact time is broadly consistent with experiments on thin supported layers of diblock poly(2-vinylpyridine)-polybutadiene copolymer, ${ }^{25}$ where the outer polybutadiene layers $\left(M_{\mathrm{w}}=38500 \mathrm{~g} / \mathrm{mol}\right.$, layer thickness $9 \AA$ ) were also allowed to interdiffuse during contact times of $0.01-500 \mathrm{~s}$ at a temperature above their bulk $T_{\mathrm{g}}$. A recent study ${ }^{45}$ on diffusion between one thin, supported, and one thicker, covering layer of poly(methyl methacrylate) $\left(M_{\mathrm{w}}\right.$
$=143000 \mathrm{~g} / \mathrm{mol}$ ) at a temperature $35{ }^{\circ} \mathrm{C}$ above $T_{\mathrm{g}}$ suggests that interdiffusion of supported layers thinner than $0.4 R_{\mathrm{g}}$ was slowed down by about 2 orders of magnitude compared to diffusion in bulk and approximately proportional to $t^{1 / 8}$, due to restricted chain conformation and to the large number of contact points between the polymer chains and the solid, supporting (silicon) surface. The slowly developing increase in adhesion observed with contact time in our experiment is thus likely the result of interdiffusion of polymer chains whose mobility across a very thin film is restricted compared to the case in the bulk. The longest contact time studied (500 s) does not necessarily constitute an upper limit for the diffusion process to continue.

In order to quantify the critical unloading rate (the onset of rate dependence), it was convenient to plot the logarithmic adhesion against logarithmic unloading rate as illustrated in Figure 5 for Fomblin Z-dol. Although this representation cannot have fundamental significance (we believe the slow rate component of the pull-off force to reflect mainly thermodynamic factors but the enhanced adhesion to reflect dissipation), we observed that the data fell empirically on straight lines, allowing a convenient consistent estimate of the critical unloading rate for each contact time. As discussed below (see also Figure 6), these critical values changed markedly with changes of the stationary contact time and differed by orders of magnitude for the different systems. Average values of the linear slopes, $B_{\text {ave }}$, obtained from graphs such as Figure 5 are tabulated for each sample in Table 2.

Contact Mechanics and Apparent Surface Energy. A common approach in adhesion experiments is to relate the measured pull-off force to the surface energy of the adsorbed layers, $\gamma$, by using the Johnson-Kendall-Roberts (JKR) theory ${ }^{10}$ of contact mechanics. Some features of this model include a finite contact area at zero external force and a separation of the surfaces from a finite contact area (with radius $=0.63 \times$ radius at zero external force $) ;{ }^{10}$ in the present experiments we observed the former but not the latter. The theory further pertains to systems whose adhesion results only from field-dependent interactions and does not treat the viscoelastic adhesion mechanisms that comprise the main subject of this study. The surface energy obtained from the pull-off force,

$$
\gamma=-F / 3 \pi R
$$

depends neither on the elastic moduli of the solid supports nor on the initial area of contact. ${ }^{10}$ It has been shown that the elastic properties and size of the surface supports used in the SFA (mica + glue layer) fall within the regime where the JKR theory is expected to be valid. ${ }^{23,46,47}$ Good agreement has been obtained between the surface energy calculated from pull-off forces and that measured with other techniques for several glassy polymer layers ${ }^{18,25}$ and close-packed surfactant monolayers, ${ }^{24,25}$ as well as for the mica surfaces themselves. ${ }^{46,47}$

In systems where attractive forces act also in a region just outside the area of molecular contact ${ }^{15,48-50}$ or where a meniscus may gather around the contact zone, ${ }^{15,46}$ the relationship between the pull-off force and surface energy is expected to be closer to

$$
\gamma=-F / 4 \pi R
$$

and the detachment then occurs when the radius of the contact area goes to zero. A brief summary of the effects of the properties of the elastic supports on the deformations and on the choice of appropriate model in each case (under the assumption of no viscoelastic contribution to the adhesion) is given in ref 51. 
For materials where viscoelastic dissipation contributes significantly to the adhesion, Greenwood and Johnson ${ }^{8}$ have predicted an increase in the effective "crack length" (effective region over which surface forces act at the edge of the contact) with increasing separation rate. The result is an increased adhesive force compared to the separation of purely elastic bodies described in the JKR model.

The surface energy, $\gamma_{0}$, for each system, calculated according to eqs 1 and 2 from the limiting $(F / R)_{0}$ at slow unloading rates, is given in Table 2. The relative error of the measurements is roughly $10 \%$ (mainly due to uncertainty in $R$ ), which exceeds the standard deviation of the $(F / R)_{0}$ values in Table 2 . We conclude that a similar value for $(F / R)_{0}$ was obtained for all four systems. Due to the viscoelastic properties of the adsorbed layers, which cause a different surface deformation at detachment than expected from the JKR theory, eq 1 likely overestimates the apparent $\gamma_{0}$, while the result obtained from eq 2 might present a lower limit.

For all four samples studied, $\gamma_{0}$ obtained from either model (Table 2) exceeds the surface energy expected for fluorocarbon polymers. Surfaces consisting of $\mathrm{CF}_{3}-$ and $-\mathrm{CF}_{2}-$ groups have a surface energy of approximately $17 \mathrm{mN} / \mathrm{m} .{ }^{52}$ More specifically, a surface energy of $17.7 \mathrm{mN} / \mathrm{m}$ for Demnum S-20 38 and $25 \mathrm{mN} / \mathrm{m}$ for our most polar sample, Z-dol, ${ }^{42}$ can be found in the literature. We also note that polyperfluoropropylene oxide has a surface energy of $19-21 \mathrm{mN} / \mathrm{m}^{26}$ Another comparison can be made with the surface energy of $13 \mathrm{mN} / \mathrm{m}$ calculated according to van der Waals - Lifshitz theory for a material with a refractive index of $1.29 .{ }^{37}$ The larger $\gamma_{0}$ measured consistently in our experiment is possibly due to some interdigitation always occurring even at the lowest unloading rates, even though the absence of observable rate dependence argues against this. It may also result from strongly perturbed chain conformations in the dried, adsorbed state relative to the surfaces of bulk samples.

As a control experiment, elsewhere we analyzed the deformations of the surfaces during separation both optically and from the output signal from the piezoelectric bimorph device. ${ }^{25}$ The surface deformations depend neither on the unloading rate nor on the contact time over the intervals studied. The enhanced adhesion measured at large unloading rates thus cannot be ascribed to differences in the deformations within the apparatus itself or the mica/glue supports, and it seems reasonable to express the observed rate dependence as an effective surface energy, $\gamma_{\text {eff }}$, which exceeds the limiting value measured at low rates. When analyzing $\gamma_{\text {eff }}$ in the discussion below, we will normalize it by $\gamma_{0}$ such that all the constants of proportionality cancel and the result amounts to a dimensionless ratio of forces, as shown in Figures 2-5.

Effects of Polar End Groups and Molecular Weight. The spreading behavior of Fomblin Z-dol as a monolayer or step with a height similar to its $R_{\mathrm{g}}$ suggests that on silicon oxide it forms an "anchored layer" with the polar end groups located at the solid surface. ${ }^{28}$ In the same experiments, the spreading of Fomblin Z-dol was strongly enhanced at $100 \%$ humidity, while the unfunctionalized polymer Fomblin $\mathrm{Z}$ was unaffected. ${ }^{28}$ This supports the concept of polar end groups being located at the solid surface, where they may be displaced by adsorbing water molecules.

Other prior studies of functionalized and unfunctionalized perfluoropoly(alkyl ether)s on solid surfaces have been based on AFM experiments. In ambient humidity, both an unfunctionalized polymer (Fomblin Z03) and one with one $-\mathrm{CH}_{2} \mathrm{OH}$ end group (Demnum SA) $\left(M_{\mathrm{w}} \approx 2000 \mathrm{~g} / \mathrm{mol}\right)$ have been found to migrate within $2 \mathrm{~h}$ to reduce in half the depth of holes (width 2-3 $\mu \mathrm{m}$, depth $20 \AA$ ) created with an AFM tip in a lubricant layer (original thickness around $20 \AA$ ) on a carbon surface. ${ }^{31}$ The mobility of Demnum SA was strongly affected by humidity. For Z-dol and a Fomblin-type polymer with $-\mathrm{COOH}$ end groups, material was not removed at ambient humidity and moderate pressures with the AFM tip. ${ }^{31}$ These layers are therefore more stable (anchored by physisorption of the polar groups) also on carbon surfaces. The apparently slower transport observed, compared to that expected from diffusion rates on silicon oxide, ${ }^{28}$ might be due to the different substrate. Another possibility is that the bottom of the hole may be quickly refilled but that the complete filling requires the much slower migration of perfluorinated polymer onto this initial layer. ${ }^{31}$ Taken together, these prior studies of various functionalized perfluorinated polymers on silicon and carbon surfaces clearly indicate that polar groups have affinity for a polar surface. Polymers of similar $M_{\mathrm{w}}$ but with different functionalized ends might thus be expected to have different affinity for the (polar) mica surfaces used in our experiment and to have their chain ends preferentially located at the solid surface.

Figure 6 shows a $\log -\log$ plot of the critical unloading rate (critical $\Delta\left(F_{\mathrm{s}} / R\right) / \Delta t$ ) vs stationary contact time before separation. To guide the eye, a line with slope $-1 / 2$ is drawn for comparison. Differences between the samples were most dramatic at the shortest contact times. By comparing Figures 2, 3A, and 3B, we find that at contact times of 0.01 or $1 \mathrm{~s}$ there is a window of unloading rates where the Demnum S-20 system displayed enhanced adhesion, while the Demnum SH-2 and Fomblin Z-dol did not. These differences vanished at the longest contact times (100 and $500 \mathrm{~s})$. The result of preferential localization of functionalized chain ends at the solid surfaces appears to be a slower initial interdigitation of the polymer layers. A slightly larger sensitivity to unloading rate in the regime of enhanced adhesion (larger $B_{\text {ave }}$ ) was observed for Fomblin Z-dol compared to Demnum S-20 and Demnum SH-2. This may possibly be due to bridging between the surfaces, since the functionalized ends of Fomblin Z-dol would have an affinity for either surface, once the interdigitation has occurred.

Differences due to chain length can be analyzed by contrasting the results for Demnum S-20 (Figure 2) with those for Fomblin Z-15 (Figure 4), though the comparison must be considered tentative because of the slightly different repeat units of the Demnum and Fomblin backbones. The thickness of both adsorbed layers is similar (cf. Table 2), but the layer of shorter chains has more chain ends per surface area and presumably faster relaxation. The greater abundance of chain ends would be expected to cause a more rapid interdiffusion in the Demnum S-20 system. In principle, this should be seen as a smaller sensitivity to contact time (i.e., the curves at different contact times might coincide), but this was not observed. In fact, the $M_{\mathrm{w}}$ of the Fomblin Z-15 sample exceeds the entanglement molecular weight of Fomblin $\mathrm{Z}$ polymers in the bulk $\left(M_{\mathrm{e}} \approx\right.$ $4000 \mathrm{~g} / \mathrm{mol}) .{ }^{53}$ Even in a thin film, this might influence the interdiffusion of layers in contact and the relaxation of chains on separation of the layers. In this context, we might rationalize that Figure 6 shows that this sample displayed the least sensitivity to contact time, but a higher sensitivity to unloading rate $\left(B_{\text {ave }}\right.$, cf. Table 2$)$ than the unfunctionalized low $M_{\mathrm{w}}$ sample, which is consistent with slower relaxations in the high $M_{\mathrm{w}}$ system.

Interpretation of Rate-Dependent Adhesion. Gent ${ }^{5}$ and others ${ }^{6,7}$ have postulated that rate-dependent adhesion may be expressed as the product of two terms: the surface energy, $\gamma$, 


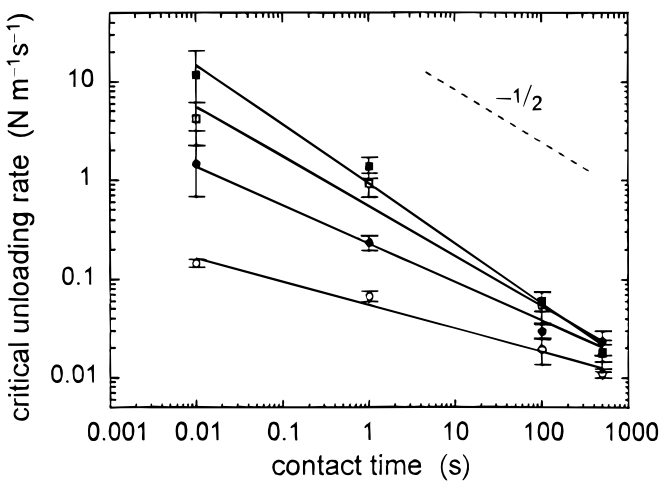

Figure 6. Critical unloading rate as a function of contact time (i.e., critical $\Delta\left(F_{\mathrm{s}} / R\right) / \Delta t$ needed to observe an increase in adhesion or pulloff force from the constant value $(F / R)_{0}$ (cf. Table 2) obtained at low

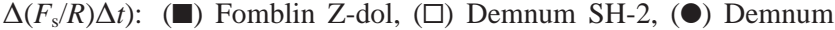
S-20, and $(\bigcirc)$ Fomblin Z-15.

and a function of viscoelastic losses within the underlying material. In the terminology of fracture mechanics, the adhesion we measure is the strain energy release rate, $G$, which can be compared to the thermodynamic work of adhesion, $W(=2 \gamma)$. The normalized excess adhesion, $(G-W) / W,{ }^{7,8}$ is equivalent to $\left(\gamma_{\text {eff }}-\gamma_{0}\right) / \gamma_{0}$. The excess adhesion has been observed in many polymer systems to grow empirically as a power law with the crack velocity (separation rate in the lateral direction) to the power 0.6. ${ }^{7}$ Greenwood and Johnson ${ }^{8}$ predicted the excess adhesion to increase as a power law of the crack velocity to the power 0.5 for a viscoelastic material whose creep compliance is either constant or linear during the time of crack propagation. The mechanical model ${ }^{8}$ was developed for bulk material but seems to be applicable to a viscoelastic interface without violating the theoretical assumptions.

The lateral separation rate at the crack tip was not measured in our experiments, but previous work on similar systems ${ }^{25}$ suggests that it was proportional to the unloading rate, i.e., to $\Delta\left(F_{\mathrm{s}} / R\right) / \Delta t$. During most of the separation, the lateral separation occurs at an average rate 10-15 times the vertical separation rate $^{25}$ (which in Figures 2-5 was varied in the range 0.003$120 \mu \mathrm{m} / \mathrm{s}$ ). It is evident from the raw data of normalized total adhesion vs unloading rate in Figures 2-4 that the excess adhesion $\left(\gamma_{\text {eff }}-\gamma_{0}\right) / \gamma_{0}$ grew more slowly than as a power law, and a plot of the excess adhesion on $\log -\log$ scales has decided curvature (not shown). In fact, the raw data in Figures 2-4 show that the excess adhesion grew approximately linearly with the logarithmic unloading rate. This may suggest that the viscoelastic relaxation function in these polymer layers does not satisfy the simple form assumed by the Greenwood-Johnson theory. Indeed, it is not unexpected to find indications of a complex viscoelastic response in ultrathin polymer layers. ${ }^{54}$

We also note that the rate-dependent regime in these systems has a velocity dependence far weaker than the simple proportionality heretofore presumed: predictions for the excess adhesion to be expected from chain pullout from an opposed layer have presumed this friction to be linearly proportional to velocity. ${ }^{20,21}$ The discrepancy might in principle arise from an inability to produce low enough crack velocity to achieve a linear viscoelastic response, but if so, the linear regime is not experimentally relevant, since effects in this regime must be overwhelmed by the larger constant term, the thermodynamic work of adhesion that was always observed when the rate was low.

We note that a similar relation between the adhesion and separation rate as for these adsorbed layers of linear, perflu- orinated polymers has been found in experiments on an elastomer cap interacting with a flat surface covered with anchored polymer chains. ${ }^{4}$ However, in the experiments on elastomer, the adhesion depended on rate even at the slowest separation rates. The difference might reflect the longer contact times $\left(1 \mathrm{~h}^{4}\right)$ in those experiments.

\section{Conclusion}

The adhesion between thin, adsorbed layers of perfluoropoly(alkyl ether)s with different chain-end functionality was found to increase approximately in proportion to the logarithmic unloading rate, above a critical unloading rate for each contact time. This behavior, which also has been observed for polybutadiene-covered surfaces, ${ }^{25}$ appears to be general for thin, adsorbed layers of polymer above its bulk $T_{\mathrm{g}}$. The preferential location of polar chain ends at the supporting solid surface was found to influence the rate of initial interdiffusion of the adsorbed layers, even though the polymer was only physisorbed, not thermally grafted. In systems of large $M_{\mathrm{w}}$ polymer, the sensitivity to unloading rate was increased, and the sensitivity to contact time lowered as a result of slower chain relaxation.

Acknowledgment. We thank A. Koike and D. Yoon for helpful discussions on the properties of the perfluorinated polymers. This work was supported by the National Science Foundation (Tribology Program), the Exxon Research and Engineering Corporation, and IFPRI (the International Fine Particle Research Institute).

\section{References and Notes}

(1) Bell, G. I. Science 1978, 200, 618

(2) Evans, E.; Ritchie, K. Biophys. J. 1997, 72, 1541.

(3) Brown, H. R. Macromolecules 1993, 26, 1666; Science 1994, 263, 1411; Faraday Discuss. 1994, 98, 47.

(4) Deruelle, M.; Tirrell, M.; Marciano, Y.; Hervet, H.; Léger, L. Faraday Discuss. 1994, 98, 55. Deruelle, M.; Léger, L.; Tirrell, M. Macromolecules 1995, 28, 7419.

(5) Gent, A. N.; Kinloch, A. J. J. Polym. Sci. A-2 1971, 9, 659. Gent, A. N.; Schultz, J. J. Adhes. 1972, 3, 281. Gent, A. N. Langmuir 1996, 12, 4492.

(6) Andrews, E. H.; Kinloch, A. J. Proc. R. Soc. London, Ser. A 1973, $332,385$.

(7) Maugis, D.; Barquins, M. J. Phys. D, Appl. Phys. 1978, 11, 1989.

(8) Greenwood, J. A.; Johnson, K. L. Philos. Mag. 1981, 43, 697.

(9) Brown, H. R. Annu. Rev. Mater. Sci. 1991, 21, 463.

(10) Johnson, K. L.; Kendall, K.; Roberts, A. D. Proc. R. Soc. London, Ser. A 1971, 324, 301.

(11) Homola, A. M.; Mate, C. M.; Street, G. B. MRS Bull. 1990, 15 (3), 45. Homola, A. M. IEEE Trans. Magn. 1996, 32, 1812.

(12) Komvopoulos, K. Wear 1996, 200, 305. Mastrangelo, C. H. Tribol. Lett. 1997, 3, 223. Tas, N.; Vogelzang, B.; Elwenspoek, M.; Legtenberg, R. In Micro/Nanotribology and Its Applications. Proceedings of the NATO Advanced Study Institute; Bhushan, B., Ed.; Kluwer: Dordrecht, 1997; pp $621-628$.

(13) Eyre, T. S.; Dutta, K. K.; Davis, F. A. Tribol. Int. 1990, 23, 11

(14) Papay, A. G. Lubr. Eng. 1983, 39, 419.

(15) Maugis, D.; Gauthier-Manuel, B. J. Adhes. Sci. Technol. 1994, 8, 1311.

(16) Creton, C.; Leibler, L. J. Polym. Sci., Polym. Phys. 1996, 34, 545.

(17) Chaudhury, M. K.; Whitesides, G. M. Langmuir 1991, 7, 1013; Science 1992, 225, 1230.

(18) Mangipudi, V.; Tirrell, M.; Pocius, A. V. J. Adhes. Sci. Technol. 1994, 8, 1251. Mangipudi, V. S.; Huang, E.; Tirrell, M.; Pocius, A. V. Macromol. Symp. 1996, 102, 131.

(19) Tirrell, M. Langmuir 1996, 12, 4548.

(20) de Gennes, P. G. J. Phys. (Paris) 1989, 50, 2551; Langmuir 1996, $12,4497$.

(21) Raphaël, E.; de Gennes, P. G. J. Phys. Chem 1992, 96, 4002. Hong Ji; de Gennes, P.-G. Macromolecules 1993, 26, 520.

(22) Israelachvili, J. N.; Adams, G. E. J. Chem. Soc., Faraday Trans. 1 1978, 74, 975 .

(23) Sridhar, I.; Johnson, K. L.; Fleck, N. A. J. Phys. D, Appl. Phys. 1997, 30, 1710 . 
(24) Chen, Y. L.; Helm, C. A.; Israelachvili, J. N. J. Phys Chem. 1991, 95, 10736.

(25) Ruths, M.; Granick, S. Langmuir 1998, 14, 1804.

(26) Novotny, V. J. J. Chem. Phys. 1990, 92, 3189.

(27) Mate, C. M. Phys. Rev. Lett. 1992, 68, 3323.

(28) Min, B. G.; Choi, J. W.; Brown, H. R.; Yoon, D. Y.; O’Connor, T. M.; Jhon, M. S. Tribol. Lett. 1995, 1, 225.

(29) O'Connor, T. M.; Back, Y. R.; Jhon, M. S.; Min, B. G.; Yoon, D. Y.; Karis, T. E. J. Appl. Phys. 1996, 79, 5788

(30) Koinkar, V. N.; Bhushan, B. J. Appl. Phys. 1996, 79, 8071; J. Vac. Sci. Technol. A 1996, 14, 2378.

(31) Dai, Q.; Vurens, G.; Luna, M.; Salmeron, M. Langmuir 1997, 13, 4401.

(32) Jones, W. R. Tribol. Trans. 1995, 38, 557. Gschwender, L.; Snyder, C. E., Jr.; Oleksiuk, M.; Koehler, M. Tribol. Trans. 1996, 39, 368.

(33) Dhinojwala, A.; Granick, S. J. Chem. Soc., Faraday Trans. 1996 92,619 .

(34) Dhinojwala, A.; Granick, S. Macromolecules 1997, 30, 1079.

(35) Israelachvili, J. N. J. Colloid Interface Sci. 1973, 44, 259.

(36) Sanguineti, A.; Guarda, P. A.; Marchionni, G.; Ajroldi, G. Polymer 1995, 36, 3697.

(37) Israelachvili, J. N. Intermolecular and Surface Forces, 2nd ed.; Academic Press: London, 1991.

(38) Manufacturer's data.
(39) Beil. 1, IV, 388.

(40) Lo Nostro, P. Adv. Colloid Interface Sci. 1995, 56, 245.

(41) Cotts, P. M. Macromolecules 1994, 27, 6487.

(42) Gui, J.; Kuo, D.; Marchon, B.; Rauch, G. C. IEEE Trans. Magn. 1997, 33, 932.

(43) de Gennes, P. G. In Microscopic Aspects of Adhesion and Lubrication; Georges, J. M., Ed.; Elsevier: Amsterdam, 1982.

(44) Jud, K.; Kausch, H. H.; Williams, J. G. J. Mater. Sci. 1981, 16 , 204.

(45) Lin, E. K.; Wu, W. L.; Satija, S. Macromolecules 1997, 30, 7224

(46) Horn, R. G.; Israelachvili, J. N.; Pribac, F. J. Colloid Interface Sci. 1987, 115,480 .

(47) Christenson, H. K. Langmuir 1996, 12, 1404

(48) Derjaguin, B. V.; Muller, V. M.; Toporov, Yu. P. J. Colloid Interface Sci. 1975, 53, 314.

(49) Muller, V. M., Yushchenko, V. S.; Derjaguin, B. V. J. Colloid Interface Sci. 1980, 77, 91; 1983, 92, 92.

(50) Pashley, M. D. Colloids Surf. 1984, 12, 69

(51) Johnson, K. L. Langmuir 1996, 12, 4510.

(52) Wu, S. In Polymer Handbook, 3rd ed.; Brandrup, J.; Immergut, E. H., Eds.; Wiley: New York 1989; p VI/413.

(53) Marchionni, G.; Ajroldi, G.; Righetti, M. C.; Pezzin, G. Polym. Commun. 1991, 32, 71.

(54) Van Alsten, J.; Granick, S. Macromolecules 1990, 23, 4856. 